\title{
Transcription factor Pax6 is expressed by astroglia after transient brain ischemia in the rat model
}

\author{
Aleksandra Steliga ${ }^{1}$, Monika Waśkow ${ }^{1}$, Zbigniew Karwacki ${ }^{2}$, Sławomir Wójcik $^{3}$, Grażyna Lietzau ${ }^{3}$, Ilona Klejbor ${ }^{1,3}$, \\ Przemysław Kowiański ${ }^{1,3}$ \\ ${ }^{1}$ Department of Health Sciences, Pomeranian University of Slupsk, Slupsk, ${ }^{2}$ Department of Neuroanesthesiology, Medical University \\ of Gdansk, Gdansk, ${ }^{3}$ Department of Anatomy and Neurobiology, Medical University of Gdansk, Gdansk, Poland
}

\begin{abstract}
Reactive astrogliosis is regarded as an universal astrocytic response to different kinds of lesions, concerned with glial fibrillary acidic protein (GFAP) up-regulation, cellular hypertrophy and proliferation. The origin of reactive and proliferating cells in the adult brain is still disputable. Persistent progenitors as well as de-differentiating adult cells of various glial lineages are regarded as possible candidates. Pax6 transcription factor is one of the characteristic markers of astroglial de-differentiation, also important for regulation of neural and glial proliferation. Various kinds of pathological stimuli evoke reactive response, differentiated in its morphological, biochemical and immunological character. The aim of this study was to assess the dynamics of astroglial morphological and proliferative response to ischemic injury. One-hour transient focal cerebral ischemia was applied to evoke the reactive astrogliosis in twenty five adult male Wistar rats. The astrocytic morphological and proliferative reactions to ischemia were studied in the period of 6 weeks by means of GFAP and Pax6 immunofluorescent staining.

A strong reactive astroglial response was observed in the cerebral cortex and striatum, manifested by GFAP and Pax6 up-regulation and astrocytic hypertrophy. Apparent morphological changes appeared within 24 hrs after ischemia. The GFAP/Pax6 colocalization was numerous and observed 24 hrs after ischemia. A characteristic spatial distribution of GFAP/Pax6 double-labelled astrocytes and Pax6 single-labelled nuclei was revealed, with the latter situated more distantly from the ischemic core. The maximal intensity of astrocytic reaction was present from the first post-ischemic week. Astroglial hypertrophic changes and proliferative reaction were more intense in the striatum than in the cerebral cortex. Our observations reveal intensive astroglial de-differentiation and proliferative response, reflected by dynamic Pax6 up-regulation within GFAP-immunoreactive astrocytes. Transient cerebral ischemia evokes strong reactive astrogliosis, which is apparently differentiated in respect to the post-ischemic period and particular brain structure.
\end{abstract}

Key words: astrocyte, astrogliosis, brain ischemia, Pax6 transcription factor, proliferation.

\section{Introduction}

Experimental results indicate that in both physiological and pathological conditions, maintaining of an adequate cerebral blood flow (CBF) is dependent on cooperation of neurons, glia and cerebral vasculature $[2,19,23]$. In the light of data published during recent years, the role of astrocytes in this process is 
still growing $[9,30,38,53]$. One of the most important features of the astrocytes is their resistance to a decreased concentration of oxygen and glucose, resulting from their characteristic metabolic properties $[16,18,44]$. This allows them to maintain regulatory functions in conditions in which neurons are unable to sustain the balance of energetic metabolism, because of a lower level of anaerobic glycolysis, higher susceptibility to excitotoxicity and lesser concentration of antioxidants. In pathological conditions, astrocytes support neurons with antioxidants and energetic metabolites, like lactate and ATP $[3,15]$.

Reactive astrogliosis is an astrocytic response to different kinds of lesions $[43,55]$. Although the universal character of astrogliosis is accepted by many authors, there is evidence that various kinds of pathological stimuli may evoke glial response, differentiated in morphological, biochemical and immunological profiles $[3,35,51]$. Changing functions of astrocytes as well as altered astrogliogenesis observed in the course of many neurological disorders, among others in the course of Down's syndrome, may be at least partially responsible for mental retardation characteristic of this illness [60]. The most important features of the astrocytic response are: GFAP up-regulation, astrocytic hypertrophy, gliotic scar formation, proliferation, and de-differentiation, which means regaining of morphological and functional features characteristic of earlier developmental stages, presumably of radial glia-like population [6-8,61].

The reactive astrogliosis and gliotic scar formation results from activation of numerous signalling pathways influencing morphological, molecular and immunological processes. The most common signalling agents influencing this reaction include neurotransmitters and gliotransmitters (ATP, glutamate, noradrenalin), reactive oxygen species (nitric oxide; $\mathrm{NO}$ ), cytokines and interleukins (IL-1 $\alpha, \mathrm{IL}-1 \beta, \mathrm{IL}-6$, IL-10), tumour necrotic factor- $\alpha$ (TNF- $\alpha$ ), tumour growth factor- $\beta$ (TGF- $\beta$ ), lipopolysaccharide, Toll-like receptor ligands, endothelin-1 and $\beta$-amyloid $[3,51]$. Reactive gliotic response to various types of lesions is also concerned with up-regulation of numerous genes encoding markers characteristic of definite stages of glial and neuronal development, like progenitor and de-differentiation markers (nestin and Pax6), migration marker (doublecortin), cell division and proliferation marker (Ki-67) [55].

Pax6 transcription factor is one of the most important elements of astroglial response to brain tis- sue injury, up-regulated by various stimuli. In physiological conditions, Pax6 plays an important role during CNS development, being characteristic of radial glia cells, responsible for neurogenesis, cells migration, brain patterning, neuronal specification, and growing of axonal projections $[40,45,49]$. Many authors regard Pax6 function as context-dependent and inhibiting cell proliferation, promoting neuronal differentiation and astrocyte maturation [46,55]. After completing development, Pax6 remains present in a relatively small group of progenitor cells, maintaining the proliferative properties and localized in the subventricular zone of lateral cerebral ventricles as well as in the subgranular zone of dentate gyrus in the hippocampal complex. However, according to some recently published data, Pax6 is expressed postnatally in neurons of amygdala, cerebellum and olfactory bulb, as well as in some astrocytes [46]. Activation by ischemia may result in re-appearing of Pax6 in the regions distant from the proliferative zones, but localized in ischemic penumbra, surrounding the necrotic core, which indicates changes of immunological characteristics of cells localized in the areas of reduced CBF. Pax6 up-regulation and astrocytic response were studied after activation by various stimuli like inflammation, brain injury, neurodegeneration and temperature injury $[46,51,55]$. However, Pax6 changes after transient cerebral ischemia are still only fragmentarily documented in the literature. The aim of this study was to perform a morphological assessment of astrocytic response and dynamics of Pax6 up-regulation after $1 \mathrm{~h}$ transient cerebral ischemia, evoked by occlusion of the middle cerebral artery in the rat.

\section{Material and methods \\ Animals and surgical procedure}

A total of 28 adult Wistar male rats (250-350 g) were used in the study. 25 animals underwent a surgical procedure and 3 animals comprised a control group. Animals were divided into groups according to the survival period ( $3 \mathrm{~h}, 24 \mathrm{~h}, 1$ week, 4 weeks, 6 weeks). The protocols were approved by the local Ethical Committee. Animal care and treatment guidelines outlined by the European Community Council Directive of 24 November 1986 (86/609/EEC) were followed.

An anaesthetic chamber was used for the induction of anaesthesia with 5 vol. \% sevoflurane (Sevorane, Abbott, UK) administered by Sigma Elite 
Vaporizer (Penlon, UK). During the surgical procedure, the concentration was reduced to 2 vol. \% sevoflurane. The animals were spontaneously ventilated with 50 vol. \% oxygen in air. The internal body temperature was controlled with a rectal probe and maintained at $37.0^{\circ} \mathrm{C}$ using a heating pad. Blood pressure, blood glucose concentration and heart rate were monitored during the surgical procedure and maintained within the normal physiological ranges throughout the whole procedure.

Transient focal cerebral ischemia was experimentally induced by occlusion of the left middle cerebral artery (tMCAO) using the intraluminal filament technique. After a midline incision in the neck, the left external carotid artery (ECA) was carefully exposed and dissected. A 20-mm length of 4-0 monofilament nylon suture (Ethilon, UK), heat-blunted at the tip, was inserted to the left external carotid artery and pushed through the internal carotid artery to occlude the origin of the middle cerebral artery for $1 \mathrm{~h}$. Subsequently, the suture was removed carefully to allow reperfusion. The ECA was ligated and the skin was closed in the standard manner. Following surgery, the rats were allowed to recover spontaneous breathing and were kept in the standard conditions, with free access to food and water. Sham-operated animals were anesthetized in an identical manner and underwent a midline cervical incision with exposition of the left common carotid artery without its incision or ligation.

\section{Immunohistochemistry}

The animals were irreversibly anesthetized with sodium pentobarbital (thiopental sodium, Biochemie $\mathrm{GmbH}$, Germany; 80 mg/kg of body weight, i.p.) and transcardially perfused with $200 \mathrm{ml}$ of $0.9 \%$ $\mathrm{NaCl}$ solution $(\mathrm{pH} 7.4)$ at room temperature, followed by $400 \mathrm{ml}$ of fixative consisting of $4 \%$ paraformaldehyde in $0.1 \mathrm{M}$ phosphate-buffered saline (PBS,

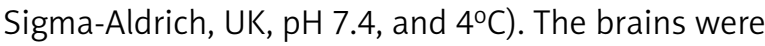
then removed and stored in the same fixative for two hours and subsequently stored in $30 \%$ sucrose in $0.1 \mathrm{M}$ PBS ( $\mathrm{pH} 7.4$ and $4^{\circ} \mathrm{C}$ ) for a minimum of $24 \mathrm{~h}$. After freezing, the brains were coronally sliced into $40 \mu \mathrm{m}$ sections on a sliding microtome.

Sections were washed in a PBS and non-specific binding was blocked by incubating the slides in blocking solution containing $5 \%$ normal goat serum (NGS)/Triton X-100/PBS for $30 \mathrm{~min}$. at room temperature. Then, they were incubated in a mixture of primary antibodies: anti-GFAP (AB 5804, Chemicon, Germany; 1 : 300) and anti-PAX 6 (AB 2237, Millipore, USA; 1 : 300) for 2 days at $4^{\circ} \mathrm{C}$. Subsequently, the sections were washed in PBS and incubated with secondary antibodies: goat anti-mouse conjugated to Alexa Fluor 488 (115-545-003, Jackson Immuno Research; USA) and goat anti-rabbit conjugated to Cy3 (Invitrogen; USA) for $1 \mathrm{~h}$ at room temperature. Slides were finally washed in PBS, dried and coverslipped with Kaiser gelatin.

The specificity of staining was verified by the omission tests. The immunohistochemical procedure was conducted as previously described, except that the primary antibodies were omitted in the presence of two secondary antibodies or secondary antibodies were omitted. In order to exclude the possibility of cross-reaction between secondary antibodies, the immunohistochemical procedure was performed with the primary antibody and non-corresponding, secondary antibody. All above-mentioned tests revealed specificity of staining and the absence of crossreaction.

\section{Confocal microscopy}

Double-labelled sections were examined with a fluorescence microscope (Eclipse E600, Nikon, Japan) equipped with a confocal imaging system (MicroRadiance, Bio-Rad, UK). In order to rule out spectral bleedthrough, the preparations were checked in non-corresponding channels. Two image analysis programs (LaserSharp 2000 v. 2.01, Bio-Rad, UK and LaserPix v. 4.0, BioRad, UK) were used to prepare the illustrations and to obtain two-dimensional colocalization plots in order to display the intensity and distribution of different coloured (green or red) pixels taken from the same optical sections within the component images. The intensities of different colours are represented on the $\mathrm{x}$ - and $\mathrm{y}$-axis, respectively. The distribution and number of differently coloured pixels appear exclusively in the cells which do not reveal the colocalization (single labelling). The yellow pixels on the plot correspond to the regions of high colocalization (double labelling).

\section{Results \\ Localization and evolution of the ischemic area in the brain}

An ischemic lesion in each case encompassed the primary and secondary somatosensory cortex, 

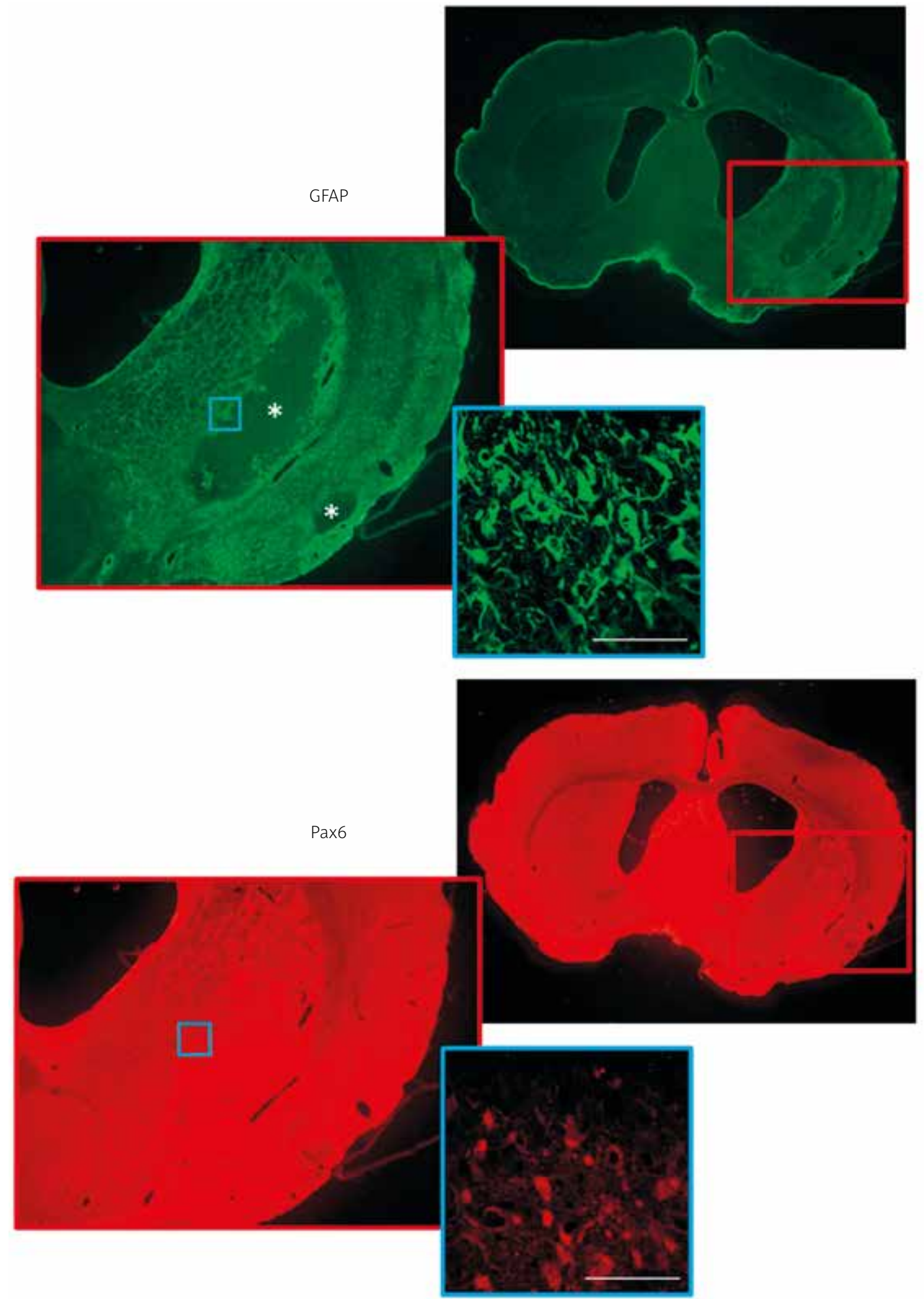

Fig. 1. Ischemic lesion in the cerebral hemisphere of the rat localized in the primary somatosensory cortex and striatum (Bregma $1.6 \mathrm{~mm}$ ), four weeks after tMCAO. Ischemic core of necrotic tissue ${ }^{*}$ ) is localized in the striatum and cerebral cortex, surrounded by gliotic scar of intensively labelled GFAP- and Pax6-ir astrocytes. (Scale bar $=50 \mu \mathrm{m})$. 
subcortical fibers of white matter and a considerable fragment of the striatum (Fig. 1). Localization and volume of the ischemic areas were comparable in all studied animals from the corresponding survival groups. An increased GFAP immunoreactivity was observed since the third hour after tMCAO. In the central part of the area corresponding to the ischemic core, gradual degeneration and cell death were observed. Successively this fragment changed into homogenous necrotic territory, without visible immunoreactive elements. The zone of increased GFAP immunoreactivity, surrounding this area changed gradually into a gliotic scar demarcating the ischemic core from a concentrically situated, hyper-reactive area of ischemic penumbra. In all studied groups, the dynamics and intensity of astroglial reaction were much more expressed in the striatum than in the cerebral cortex.

\section{Astrocytic activation in course of transient cerebral ischemia}

The morphological features of astrocytic response in the cerebral cortex and striatum were concerned with slightly up-regulated although inhomogeneous GFAP immunoreactivity (-ir), presence of hypertrophic cell bodies with dilated proximal fragments of stem branches (Figs. 2 and 3). GFAP-ir thin, ramified processes and fibers formed a delicate network. Initially ( $3 \mathrm{hrs}$ after tMCAO), GFAP-ir cell bodies, with proximal fragments of processes were rarely distributed forming distinguished spatial non-overlapping domains. This regular arrangement was disrupted with development of reactive astrogliosis at $24 \mathrm{~h}$ time point after tMCAO. A pronounced GFAP up-regulation within hypertrophic cell bodies and thick initial fragments of ramified processes were clearly distinguished. The disruption of spatial organization of astrocytic domains was present at this stage of reactive gliosis.

In all studied groups, the intensity of astroglial response was higher in the striatum than in the cerebral cortex. The morphological features of astrocytic reaction increased gradually and reached the highest level after the first week of reperfusion. The gliotic scar consisting of a dense network of GFAP-ir fibers appeared after the first week and was totally developed at the fourth week after tMCAO, indicating the severe character of astroglial reaction. Later, the intensity of astroglial response remained stabilized until the end of observation.

\section{Astrocytic de-differentiation in the ischemic area}

Pax6-ir was observed within cellular nuclei localized in the cerebral cortex, striatum and subcortical white matter fibers in the ischemic region starting from $24 \mathrm{~h}$ after initiating the ischemia (Figs. 2 and 3). Since that time GFAP/Pax6 colocalization was present until the end of observation. The proportion of GFAP/Pax6 double-labelled astrocytes increased gradually and reached the highest level at the fourth week after $\mathrm{tMCAO}$.

Double-staining image analysis using two-dimensional co-localization plots for presentation of the staining intensity and colocalization revealed prevalence of green signal (for GFAP-ir) in all studied groups in the cortex and striatum (Fig. 4). Red signal (for Pax6-ir) appeared at $24 \mathrm{~h}$ after tMCAO in both structures and increased until the end of the study. Yellow signal (for GFAP/Pax6 colocalization) appeared at $24 \mathrm{~h}$ in both structures and increased systematically in the striatum, whereas was most intense after four weeks in the cortex.

Although the majority of GFAP-ir astrocytes in the cortex and striatum revealed Pax6-ir nuclei, GFAPand Pax6-single labelled cells were also present. Characteristically, Pax6-ir single-labelled nuclei were localized more peripherally from the ischemic core and dense concentric GFAP/Pax6-ir astroglial layer.

\section{Discussion}

\section{Morphology of astrocytic response to transient ischemia}

Transient cerebral ischemia is a strong stimulating factor initiating reactive astrogliosis. Morphological changes like increasing GFAP immunoreactivity, astrocytic hypertrophy and disruption of the astroglial microdomains, increased gradually and were apparent from $24 \mathrm{hrs}$ after tMCAO. Maximal intensity of astrocytic reaction in our model was observed after one week of reperfusion and was maintained until the end of observation. Gliotic scar was formed at the same time, demarcating centrally localized necrotic core. Morphological features, intensity and dynamics of gliotic response, reported here in the ischemic model, correspond with gradual development of mild, moderate and severe stages of reactive astrogliosis according to Sofroniew division [51].

GFAP up-regulation is a characteristic and useful marker of astrocytic response to various kinds of 

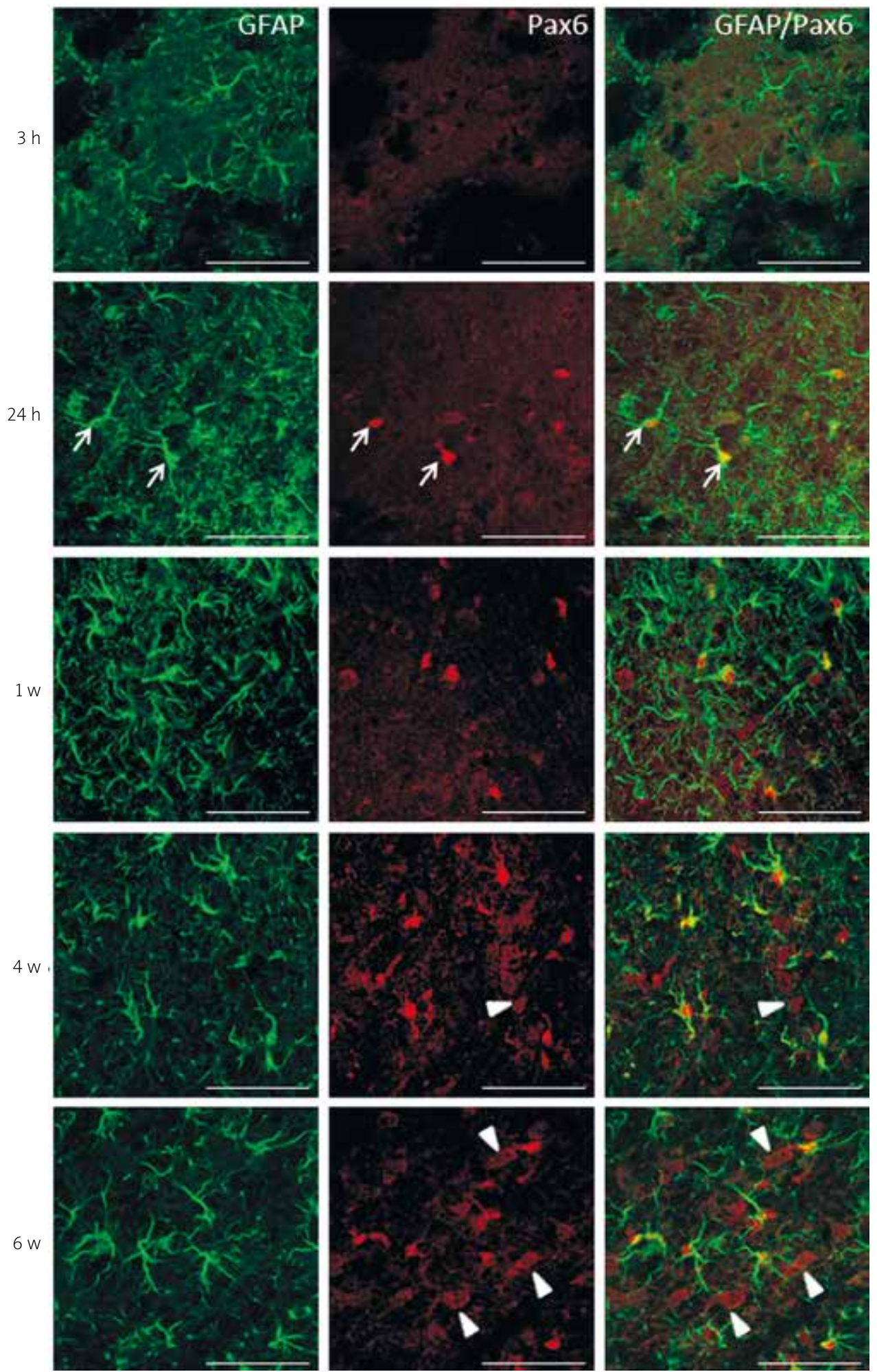

Fig. 2. Reactive astrogliosis in the cerebral cortex of the rat in response to transient focal ischemia. GFAP up-regulation and astrocytic hypertrophy occur at $24 \mathrm{~h}$ time point after tMCAO and increase gradually during the period of study. Astrocytic de-differentiation assessed on the basis of Pax 6 up-regulation in the cellular nuclei is present from $24 \mathrm{~h}$ after tMCAO. GFAP/Pax6 colocalization in reactive astrocytes is frequently observed (arrows), although Pax6 single-labelled nuclei (arrowheads) are also present, which may be explained by de-differentiation and proliferative activity of other cellular lineages. (Scale bar $=50 \mu \mathrm{m}$ ). 

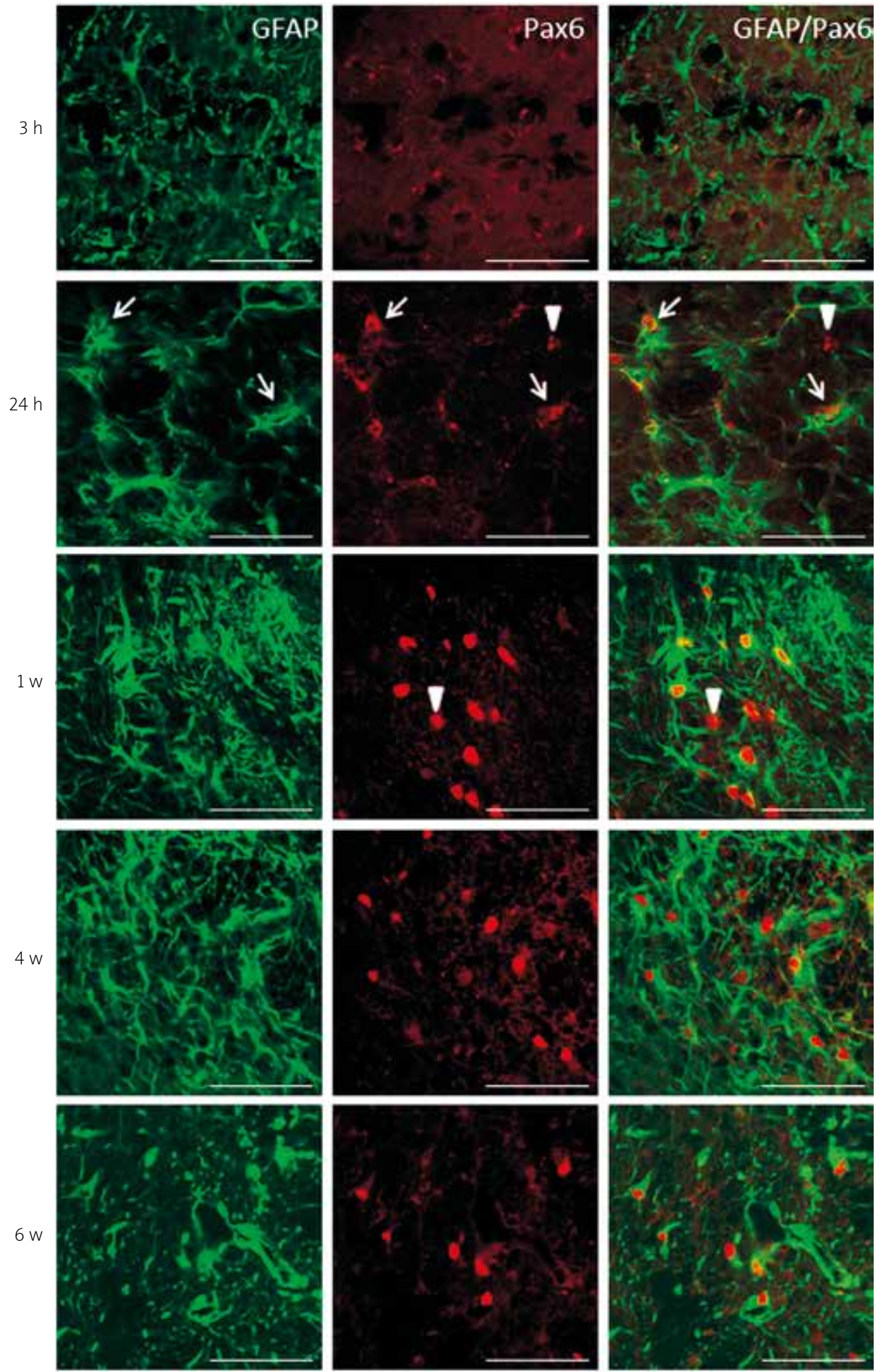

Fig. 3. Reactive astrogliosis in the striatum of the rat in response to transient focal ischemia. GFAP up-regulation and astrocytic hypertrophy start to appear at $24 \mathrm{~h}$ after $\mathrm{AMCAO}$ and reveal maximal intensity from the first to the fourth week after tMCAO. Pax6-ir nuclei appear at $24 \mathrm{~h}$ after initiating of the ischemia, revealing de-differentiation predominantly of reactive astroglia (GFAP/Pax6 colocalization; arrows). However, similarly to the cerebral cortex, Pax6 single-labelled nuclei are also detectable (arrowheads), which indicates proliferative response of other cellular subpopulations. Astroglial response to ischemic lesion in the striatum is significantly more intense than in the cerebral cortex. (Scale bar $=50 \mu \mathrm{m})$. 


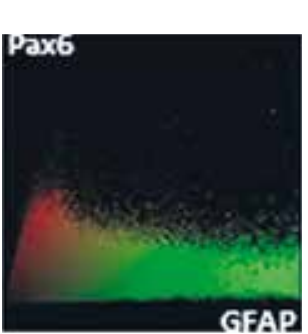

$3 \mathrm{~h}$

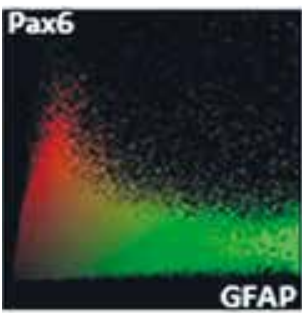

$3 \mathrm{~h}$

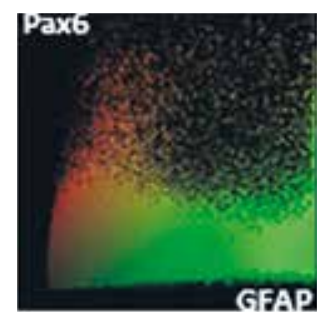

$24 \mathrm{~h}$

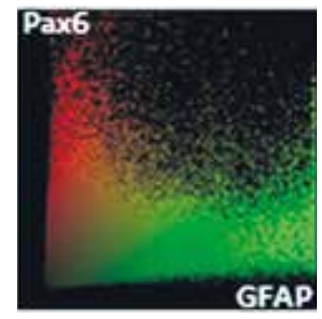

$24 \mathrm{~h}$

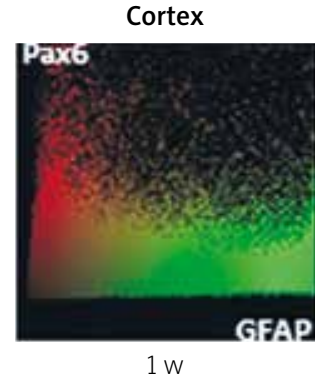

$1 \mathrm{w}$

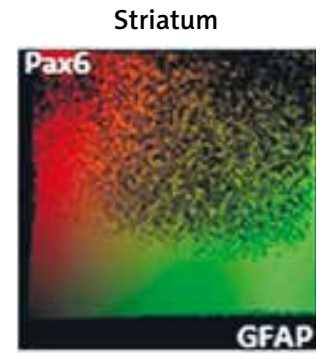

$1 \mathrm{w}$

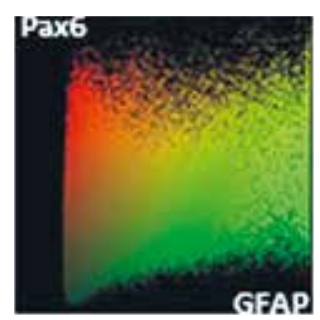

$4 \mathrm{w}$

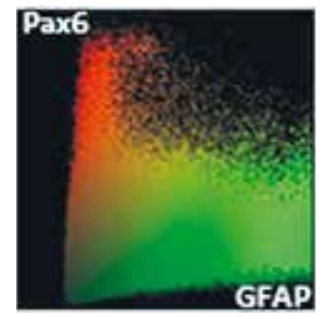

$4 \mathrm{w}$
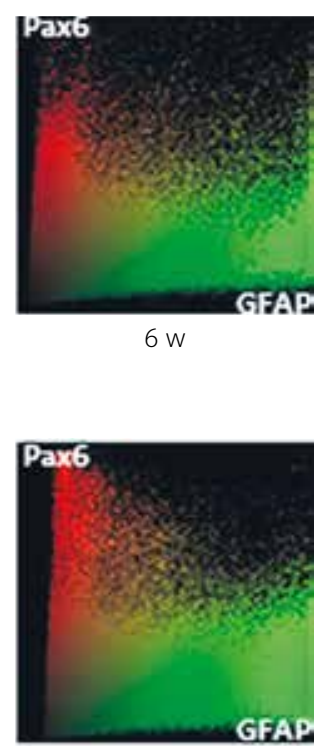

$6 \mathrm{w}$

Fig. 4. Double-staining image analysis using two-dimensional co-localization plots presenting the intensity and localization of differently coloured pixels taken from the same optical sections within the component images. The yellow pixels on the plot correspond to the high degree of co-localization, whereas green (for GFAP staining) and red (for Pax6 staining) pixels represent the single labelled structures.

lesions. However, it must be stressed that this protein does not occur in equal concentration in all astrocytic cells [51]. In astroglia remote from the lesion, or non-reactive cells of healthy tissue, GFAP may not be detected. Moreover, GFAP immunostaining can give an incorrect impression of an activated territory, considerably underestimating the extent of astrocytic branching. This protein is almost completely absent in small branching and very often only slightly staining the astrocytic cell bodies. Consequently, the distribution of GFAP within the astroglial cell is uneven and does not reflect the detailed and complete morphology. After evoked transient ischemia we observed strong up-regulation of GFAP in reactive astroglia of the territory supplied by the occluded MCA. The intensity of immunofluorescence was apparently increased in comparison to the intensity of staining in the regions of unaffected brain tissue.

\section{Proliferative activity of astrocytes in response to transient ischemia}

Our results point to rapid and intensive up-regulation of Pax6 transcription factor in the astrocytes localized within the ischemic region. Colocalization of GFAP/Pax6 was revealed starting from $24 \mathrm{~h}$ after initiating the ischemia and continued during the whole observation period. Apart from GFAP/Pax6 double-labelled cells we have also observed Pax6single-labelled nuclei and GFAP-single-labelled cell bodies, what suggests in favour of 1) Pax6 occurrence in other glial subpopulations, as well as in neurons of this area and 2) occurrence of astrocytes not responding with de-differentiation and proliferative reaction. Our preliminary and unpublished observations have revealed NG2/Pax6 colocalization in the same model of transient brain ischemia. In summary, these results point to general intensive proliferative activity in the ischemic region, confirm the prevalent role of astrocytes in this process but do not preclude the possible contribution of the other cellular components in proliferative response [24,25]. It still remains an unanswered question which other cellular populations start to de-differentiate and proliferate in conditions created by transient brain ischemia. On the basis of literature review, possible candidates revealing proliferative potential in response to various types of stimulating factors may be considered to include progenitor cells of the subventricular zone (SVZ) [11,20], NG2 cells [39,56], Olig2 cells [54] and resident astrocytes [6,58,59]. 
It seems to be important that GFAP/Pax6 colocalization is almost undetectable during the early postischemic period ( $3 \mathrm{hrs}$ ) and becomes much more frequently observed at 24 hrs after IMCAO, increasing further during the study period. This may be explained by dynamic de-differentiation of resident astrocytes, which gain immunological and functional characteristics of neural progenitor cells $[1,27,31]$.

As it has been mentioned by other authors, reactive astrogliosis occurs in a spatially segregated, concentric manner, which is reflected by localization of different kinds of cell types, immunoreactive to NG2, Olig2, GFAP and vimentin [29]. This corresponds with our observation concerned with differentiated localization of GFAP/Pax6-double labelled cells and Pax6-single labelled nuclei in various regions of the ischemic area. Consequently, this may indicate 1) a regionally specific character of glial response to ischemic injury, 2) differentiated potential of proliferative reaction resulting from the energetic metabolism impairment in different regions of penumbra and 3) possibly not only astroglial origin of reacting and proliferating cells.

While examining the time-course of changes in glial cells of the ischemic area, we found that maximal intensity of astroglial response was present after the first week after tMCAO. Dynamics of astroglial reaction observed in our model is generally similar to that reported in the course of other types of brain injury $[29,43]$.

\section{Comparison of the reactive astrogliosis in the cerebral cortex and striatum}

Comparing the intensity of astrocytic morphological reaction, as well as de-differentiation and proliferative response, assessed on the basis of Pax6 immunoreactivity, we observed much more expressed morphological features of reactive astrogliosis in the striatum than in the cerebral cortex, during the whole studied period. This apparent difference may be explained with various anatomical sources of vascular supply by deep perforating, subcortical branches and terminal cortical branches of the middle cerebral artery, respectively [22]. It has been proved that various brain regions are characterized by differentiated density of capillary vessels [10], which results in unequal levels of CBF reduction, adjusted to the metabolic demands. In pathological circumstances, it may explain different susceptibility of these regions to cerebral ischemia [15]. Another explanation may be concerned with different susceptibility of protoplasmic and fibrous astrocytes to ischemic conditions. There is evidence that protoplasmic astrocytes, which are more numerous in the cortical regions, are less resistant to the decreased oxygen and glucose concentration than fibrous astrocytes, localized in the white matter and some subcortical regions $[3,35]$.

\section{Functional significance of reactive gliosis and astrocytic proliferation}

The reactive astrogliosis reveals both detrimental and neuroprotective roles in the course of pathological processes. A decreasing concentration of oxygen and glucose in the course of cerebral ischemia results in energetic metabolism impairment $[3,51]$. Decreasing metabolic activity is reflected by inhibition of glutamate uptake, and rising excitotoxicity $[4,41,53]$. Meanwhile, the production of neurotoxic reactive oxygen species (e.g. NO) increases in astrocytes, and through diffusion within the surrounding area of ischemic core and penumbra, significantly impairs neuronal survival potential [17,32,33]. Inflammatory reaction is triggered by cytokines production stimulated by NF- $\kappa B$ transcription factor $[14,52,53]$. Changes of aquaporin-4 activity can compromise the blood-brain-barrier integrity, which results in cytotoxic oedema. Later, inhibition of axonal regeneration through the gliotic scar may significantly decrease the functional recovery after cerebral ischemia.

The protective function of reactive astrocytes results from their ability of excitotoxic glutamate re-uptake $[12,26]$, inhibition of tissue plasminogen activator-1 [34], production of antioxidants like glutathione, superoxide dismutase and ascorbate [3], amyloid-beta degradation [57], re-establishing of the blood-brain-barrier continuity [13, 36], maintaining of the ionic concentration and extracellular fluid volume, protection against epileptic seizures concerned with GABA production and maintaining of its concentration [47], stimulation of gliogenesis $[21,37,48]$ and neurogenesis $[5,28,42]$. Gliotic scar can limit spreading of inflammatory cytokines and proapoptotic agents from the damaged area protecting the surrounding tissue from the consequences of the pathological process $[43,50]$.

In summary, transient cerebral ischemia evokes strong reactive astrogliosis with a prominent prolif- 
erative component. Pax6 transcription factor up-regulation in GFAP-ir astrocytes distinctly underscores their proliferative potential in the course of cerebral ischemia. The intensity of reactive astrogliosis is apparently differentiated in respect to the post-ischemic time period and brain structure.

\section{Acknowledgements}

The authors kindly appreciate technical assistance of Dr Beata Domaradzka-Pytel in the preparation of this manuscript.

\section{References}

1. Alvarez-Buylla A, Seri B, Doetsch F. Identification of neural stem cells in the adult vertebrate brain. Glia 2002; 57: 751-758.

2. Araque A, Parpura V, Sanzgiri RP, Haydon PG. Tripartite synapses: glia, the unacknowledged partner. Trends Neurosci 1999; 22: 208-215.

3. Barreto GE, Gonzalez J, Torres Y, Morales L. Astrocytic-neuronal crosstalk: implications for neuroprotection from brain injury. Neurosci Res 2011; 71: 107-113.

4. Bastide M, Ouk T, Plaisier F, Pétrault O, Stolc S, Bordet R. Neurogliovascular unit after cerebral ischemia: is the vascular wall a pharmacological target. Psychoneuroendocrinology 2007; 32: 36-39.

5. Berninger B, Costa MR, Koch U, Schroeder T, Sutor B, Grothe B, Götz M. Functional properties of neurons derived from in vitro reprogrammed postnatal astroglia. J Neurosci 2007; 27: 8654-8664.

6. Buffo A, Rite I, Tripathi P, Lepier A, Colak D, Horn AP, Mori T, Götz M. Origin and progeny of reactive gliosis: a source of multipotent cells in the injured brain. Proc Natl Acad Sci USA 2008; 105: 3581-3586.

7. Buffo A, Rolando C, Ceruti S. Astrocytes in the damaged brain: molecular and cellular insights into their reactive response and healing potential. Biochem Pharmacol 2010; 79: 77-89.

8. Burns KA, Murphy B, Danzer SC, Kuan CY. Developmental and post-injury cortical gliogenesis: a genetic fate-mapping study with Nestin-CreER mice. Glia 2009; 57: 1115-1129.

9. Carmignoto G, Gómez-Gonzalo M. The contribution of astrocyte signalling to neurovascular coupling. Brain Res Rev 2010; 63: 138-148.

10. Cavaglia M, Dombrowski SM, Drazba J, Vasanji A, Bokesch PM, Janigro D. Regional variation in brain capillary density and vascular response to ischemia. Brain Res 2001; 910: 81-93.

11. Chojnacki AK, Mak GK, Weiss S. Identity crisis for adult periventricular neural stem cells: subventricular zone astrocytes, ependymal cells or both? Nat Rev Neurosci 2009; 10: 153-163.

12. David Y, Cacheaux LP, Ivens S, Lapilover E, Heinemann U, Kaufer D, Friedman A Astrocytic dysfunction in epileptogenesis: consequence of altered potassium and glutamate homeostasis? J Neurosci 2009; 29: 10588-10599.

13. del Zoppo GJ. Virchow's triad: the vascular basis of cerebral injury. Rev Neurol Dis 2008; 5: 12-21.
14. del Zoppo GJ. The neurovascular unit in the setting of stroke. J Intern Med 2010; 267: 156-171.

15. Figley CR, Stroman PW. The roles of astrocytes and astrocyte activity in neurometabolism, neurovascular coupling, and the production of functional neuroimaging signals. Eur J Neurosci 2011; 33: 577-588.

16. Filosa JA, Bonev AD, Nelson, MT. Calcium dynamics in cortical astrocytes and arterioles during neurovascular coupling. Circ Res 2004; 95: 73-81.

17. Girouard H, ladecola C. Neurovascular coupling in the normal brain and in hypertension, stroke, and Alzheimer disease. J Appl Physiol 2006; 1001: 328-335.

18. Gordon GR, Choi B, Rungta RL, Ellis-Davies GC, MacVicar BA. Brain metabolism dictates the polarity of astrocyte control over arterioles. Nature 2008; 456: 745-749.

19. Gordon GR, Mulligan SJ, MacVicar BA. Astrocyte control of the cerebrovasculature. Glia 2007; 55: 1214-1221.

20. Gould E. How widespread is adult neurogenesis in mammals? Nat Rev Neurosci 2007; 8: 481-488.

21. Gubert F, Zaverucha-do-Valle C, Pimentel-Coelho PM, MendezOtero R, Santiago MF. Radial glia-like cells persist in the adult rat brain. Brain Res 2009; 1258: 43-52.

22. Hamel E. Perivascular nerves and the regulation of cerebrovascular tone. J Appl Physiol 2006; 100: 1059-1064.

23. Haydon PG, Carmignoto G. Astrocyte control of synaptic transmission and neurovascular coupling. Physiol Rev 2006; 86: 1009-1031.

24. Heinrich C, Blum R, Gascón S, Masserdotti G, Tripathi P, Sánchez R, Tiedt S, Schroeder T, Götz M, Berninger B. Directing astroglia from the cerebral cortex into subtype specific functional neurons. PLoS Biol 2010; 8: e1000373.

25. Heins N, Malatesta P, Cecconi F, Nakafuku M, Tucker KL, Hack MA, Chapouton P, Barde YA, Götz M. Glial cells generate neurons: the role of the transcription factor Pax6. Nat Neurosci 2002; 5: 308-315.

26. Héja L, Barabás P, Nyitrai G, Kékesi KA, Lasztóczi B, Toke O, Tárkányi G, Madsen K, Schousboe A, Dobolyi A, Palkovits M, Kardos J. Glutamate uptake triggers transporter-mediated GABA release from astrocytes. PLoS One 2009; 4: 7153.

27. Ihrie RA, Alvarez-Buylla A. Lake-front property: a unique germinal niche by the lateral ventricles of the adult brain. Neuron 2011; 70: 674-686.

28. Jin K, Sun Y, Xie L, Peel A, Mao XO, Batteur S, Greenberg DA. Directed migration of neuronal precursors into the ischemic cerebral cortex and striatum. Mol Cell Neurosci 2003; 24: 171-189.

29. Kim WR, Kim JY, Moon Y, Kim HJ, Kim H, Sun W. Regional difference of reactive astrogliosis following traumatic brain injury revealed by hGFAP-GFP transgenic mice. Neurosci Lett 2012; 513: 155-159.

30. Kowiański P, Lietzau G, Steliga A, Waśkow M, Moryś J. Astrocytic contribution to neurovascular coupling - still more questions than answers? Neurosci Res 2013; 75: 171-83.

31. Kriegstein A, Alvarez-Buylla A. The glial nature of embryonic and adult neural stem cells. Annu Rev Neurosci 2009; 32: 149-184.

32. Lauritzen M, Dreier JP, Fabricius M, Hartings JA, Graf R, Strong AJ. Clinical relevance of cortical spreading depression in neurolo- 
gical disorders: migraine, malignant stroke, subarachnoid and intracranial hemorrhage, and traumatic brain injury. J Cereb Blood Flow Metab 2011; 31: 17-35.

33. Lauritzen M, Mathiesen C, Schaefer K, Thomsen KJ. Neurona inhibition and excitation, and the dichotomic control of brain hemodynamic and oxygen responses. Neuroimage 2012; 62: 1040-1050.

34. Li L, Lundkvist A, Andersson D, Wilhelmsson U, Nagai N, Pardo AC, Nodin C, Ståhlberg A, Aprico K, Larsson K, Yabe T, Moons L, Fotheringham A, Davies I, Carmeliet P, Schwartz JP, Pekna M, Kubista M, Blomstrand F, Maragakis N, Nilsson M, Pekny M. Protective role of reactive astrocytes in brain ischemia. J Cereb Blood Flow Metab 2008; 28: 468-481.

35. Lukaszevicz AC, Sampaïo N, Guégan C, Benchoua A, Couriaud C, Chevalier E, Sola B, Lacombe P, Onténiente B. High sensitivity of protoplasmic cortical astroglia to focal ischemia. J Cereb Blood Flow Metab 2002; 22: 289-298.

36. Milner R, Hung S, Wang X, Berg GI, Spatz M, del Zoppo GJ. Responses of endothelial cell and astrocyte matrix-integrin receptors to ischemia mimic those observed in the neurovascular unit. Stroke 2008; 39: 191-197.

37. Morrens J, Van Den Broeck W, Kempermann G. Glial cells in adult neurogenesis. Glia 2012; 60: 159-174.

38. Neuwelt EA, Bauer B, Fahlke C, Fricker G, ladecola C, Janigro D, Leybaert L, Molnár Z, O’Donnell ME, Povlishock JT, Saunders NR, Sharp F, Stanimirovic D, Watts RJ, Drewes LR. Engaging neuroscience to advance translational research in brain barrier biology. Nat Rev Neurosci 2011; 12: 169-182.

39. Nishiyama A, Komitova M, Suzuki R, Zhu X. Polydendrocytes (NG2 cells): multifunctional cells with lineage plasticity. Nat Rev Neurosci 2009; 10: 9-22.

40. Osumi N. The role of Pax6 in brain patterning. Tohoku J Exp Med 2001; 193: 163-174.

41. Ouyang YB, Voloboueva LA, Xu LJ, Giffard RG. Selective dys function of hippocampal CA1 astrocytes contributes to delayed neuronal damage after transient forebrain ischemia. J Neurosci 2007; 27: 4253-4260.

42. Parent JM, Vexler ZS, Gong C, Derugin N, Ferriero DM. Rat forebrain neurogenesis and striatal neuron replacement after focal stroke. Ann Neurol 2002; 52: 802-813.

43. Pekny M, Nilsson M. Astrocyte activation and reactive gliosis. Glia 2005; 50: 427-434

44. Petzold GC, Murthy VN. Role of astrocytes in neurovascular coupling. Neuron 2011; 71: 782-797.

45. Prosser J, Heyningen V van. PAX6 mutations reviewed. Hum Mutat 1998; 11: 93-108.

46. Sakurai K, Osumi N. The neurogenesis-controlling factor, Pax6, inhibits proliferation and promotes maturation in murine astrocytes. J Neurosci 2008; 28: 4604-4612.

47. Schousboe A, Sarup A, Bak LK, Waagepetersen HS, Larsson OM Role of astrocytic transport processes in glutamatergic and GABAergic neurotransmission. Neurochem Int 2004; 45: 521-527.

48. Simon C, Götz M, Dimou L. Progenitors in the adult cerebral cortex: cell cycle properties and regulation by physiological stimuli and injury. Glia 2011; 59: 869-881.

49. Simpson TI, Price DJ. Pax6; a pleiotropic player in development. Bioessays 2002; 24: 1041-1051.
50. Sofroniew MV. Molecular dissection of reactive astrogliosis and glial scar formation. Trends Neurosci 2009; 32: 638-647.

51. Sofroniew MV, Vinters HV. Astrocytes: biology and pathology. Acta Neuropathol 2010; 119: 7-35.

52. Stanimirovic DB, Satoh K. Inflammatory mediators of cerebral endothelium: a role in ischemic brain inflammation. Brain Pathol 2000; 10: 113-126.

53. Stanimirovic DB, Friedman A. Pathophysiology of the neurovascular unit: disease cause or consequence? J Cereb Blood Flow Metab 2012; 32: 1207-1221.

54. Tatsumi K, Takebayashi H, Manabe T, Tanaka KF, Makinodan M, Yamauchi T, Makinodan E, Matsuyoshi H, Okuda H, Ikenaka K, Wanaka A. Genetic fate mapping of Olig2 progenitors in the injured adult cerebral cortex reveals preferential differentiation into astrocytes. J Neurosci Res 2008; 86: 3494-3502.

55. Wachter B, Schürger S, Rolinger J, von Ameln-Mayerhofer A, Berg D, Wagner HJ, Kueppers E. Effect of 6-hydroxydopamine (6-OHDA) on proliferation of glial cells in the rat cortex and striatum: evidence for de-differentiation of resident astrocytes. Cell Tissue Res 2010; 342: 147-160.

56. Wang A, He BP. Characteristics and functions of NG2 cells in normal brain and neuropathology. Neurol Res 2009; 31: 144-150.

57. Wyss-Coray T, Loike JD, Brionne TC, Lu E, Anankov R, Yan F, Silverstein SC, Husemann J. Adult mouse astrocytes degrade amyloid-beta in vitro and in situ. Nat Med 2003; 9: 453-457.

58. Yang H, Cheng XP, Li JW, Yao Q, Ju G. De-differentiation response of cultured astrocytes to injury induced by scratch or conditioned culture medium of scratch-insulted astrocytes. Cell Mol Neurobiol 2009; 29: 455-473.

59. Yu T, Cao G, Feng L. Low temperature induced dedifferentiation of astrocytes. J Cell Biochem 2006; 99: 1096-1107.

60. Zdaniuk G, Wierzba-Bobrowicz T, Szpak GM, Stępień T. Astroglia disturbances during development of the central nervous system in fetuses with Down's syndrome. Folia Neuropathol 2011; 49: 109-114.

61. Zhao JW, Raha-Chowdhury R, Fawcett JW, Watts C. Astrocytes and oligodendrocytes can be generated from NG2+ progenitors after acute brain injury: intracellular localization of oligodendrocyte transcription factor 2 is associated with their fate choice. Eur J Neurosci 2009; 29: 1853-1869. 